\title{
Accurate millimetre and submillimetre rest frequencies for cis- and trans-dithioformic acid, $\mathrm{HCSSH}^{\star}$
}

\author{
D. Prudenzano ${ }^{1}$, J. Laas ${ }^{1}$, L. Bizzocchi ${ }^{1}$, V. Lattanzi ${ }^{1}$, C. Endres ${ }^{1}$, B. M. Giuliano ${ }^{1}$, \\ S. Spezzano ${ }^{1}$, M. E. Palumbo ${ }^{2}$, and P. Caselli ${ }^{1}$
${ }^{1}$ Center for Astrochemical Studies, Max-Planck-Institut für extraterrestrische Physik, Gießenbachstraße 1, 85748 Garching, Germany e-mail: prudenzano@mpe.mpg.de
2 INAF-Osservatorio Astrofisico di Catania, 95123 Catania, Italy
e-mail: mepalumbo@oact.inaf.it

Received 1 December 2017 / Accepted 21 December 2017

\begin{abstract}
Context. A better understanding of sulphur chemistry is needed to solve the interstellar sulphur depletion problem. A way to achieve this goal is to study new S-bearing molecules in the laboratory, obtaining accurate rest frequencies for an astronomical search. We focus on dithioformic acid, HCSSH, which is the sulphur analogue of formic acid.

Aims. The aim of this study is to provide an accurate line list of the two HCSSH trans and cis isomers in their electronic ground state and a comprehensive centrifugal distortion analysis with an extension of measurements in the millimetre and submillimetre range.

Methods. We studied the two isomers in the laboratory using an absorption spectrometer employing the frequency-modulation technique. The molecules were produced directly within a free-space cell by glow discharge of a gas mixture. We measured lines belonging to the electronic ground state up to $478 \mathrm{GHz}$, with a total number of 204 and 139 new rotational transitions, respectively, for trans and cis isomers. The final dataset also includes lines in the centimetre range available from literature.

Results. The extension of the measurements in the $\mathrm{mm}$ and submm range lead to an accurate set of rotational and centrifugal distortion parameters. This allows us to predict frequencies with estimated uncertainties as low as $5 \mathrm{kHz}$ at $1 \mathrm{~mm}$ wavelength. Hence, the new dataset provided by this study can be used for astronomical search.
\end{abstract}

Key words. molecular data - methods: laboratory: molecular - techniques: spectroscopic - radio lines: ISM - ISM: molecules

\section{Introduction}

Sulphur chemistry has attracted interest since the early 70 s, when the first S-bearing molecule, CS, was detected in the interstellar medium (Penzias et al. 1971), followed by observations of other sulphur species in the same decade, such as OCS, $\mathrm{H}_{2} \mathrm{~S}$, and $\mathrm{CH}_{3} \mathrm{SH}$ (Jefferts et al. 1971, Thaddeus et al. 1972, Linke et al. 1979 and references therein). In diffuse clouds sulphur is mainly found in the gas phase in the form of $\mathrm{S}^{+}$and has cosmic abundances of $\sim 10^{-5}$ with respect to the H nuclei (Jenkins 1987; Savage \& Sembach 1996). However, to reproduce the observed values of S-bearing molecules in dark clouds (Oppenheimer \& Dalgarno 1974; Tieftrunk et al. 1994), chemical models typically assume that sulphur is highly depleted, using an initial elemental abundance of $8 \times 10^{-8}$ (Wakelam \& Herbst 2008). A possible explanation of this problem is given by Ruffle et al. (1999), who proposed that during collapse of translucent gas, sulphur tends to freeze-out more than other elements in dark clouds, due to its predominant ionized form, $\mathrm{S}^{+}$, and thus it is subject to strong electrostatic attraction to negatively charged grains.

Many studies have discussed the problem and some have suggested that most of the missing sulphur in dense regions could be trapped in the solid phase as either polysulphanes, having the formula $\mathrm{H}_{x} \mathrm{~S}_{y}$ (Jiménez-Escobar \& Muñoz Caro 2011;

\footnotetext{
* Frequency lists are only available at the CDS via anonymous ftp to cdsarc.u-strasbg.fr $(130.79 .128 .5)$ or via http://cdsarc.u-strasbg.fr/viz-bin/qcat?]/A+A/612/A56
}

Druard \& Wakelam 2012), or refractory sulphur polymers, such as $S_{8}$ (Wakelam et al. 2004, 2005). However, the main reservoirs of solid sulphur are still unknown. Some laboratory studies, focussing on dust and ice analogues, propose carbon disulfide, $\mathrm{CS}_{2}$, as a major sink of sulphur (Ferrante et al. 2008; Garozzo et al. 2010; Jiménez-Escobar et al. 2014). Recently, $\mathrm{CS}_{2}$ has been found in the comas of some comets through the identification of its emission spectra in the UV and visible region (Jackson et al. 2004) or in situ by ROSINA (Calmonte et al. 2016). In particular, Calmonte et al. (2016) have found that the amount of $\mathrm{CS}_{2}$ in the coma of 67P/Churyumov-Gerasimenko accounted for the $0.35 \%$ of the total sulphur content, resulting more abundant than thioformaldehyde, $\mathrm{H}_{2} \mathrm{CS}(0.14 \%)$, a well-known S-bearing molecule, detected for the first time in SgrB2 by Sinclair et al. (1973). Nevertheless, carbon disulfide is still undetected in interstellar ices. Furthermore, in warm and shocked regions, where this compound could be transferred in the gas phase as a result of evaporation and sputtering of the grains, its detection by means of radio telescopes is unfeasible because it has no permanent dipole moment. Therefore other S-bearing molecules must be sought.

Recently, many steps have been taken towards improving our understanding of interstellar sulphur chemistry, as highlighted by the detection of new S-bearing compounds, such as $\mathrm{S}_{3}, \mathrm{~S}_{4}$, and ethyl mercaptan $\left(\mathrm{CH}_{3} \mathrm{CH}_{2} \mathrm{SH}\right)$ in the comas of 67P/ChuryumovGerasimenko (Calmonte et al. 2016) and a tentative detection of $\mathrm{CH}_{3} \mathrm{CH}_{2} \mathrm{SH}$ in Orion KL (Kolesniková et al. 2014). Moreover, chemical models have been significantly improved through 

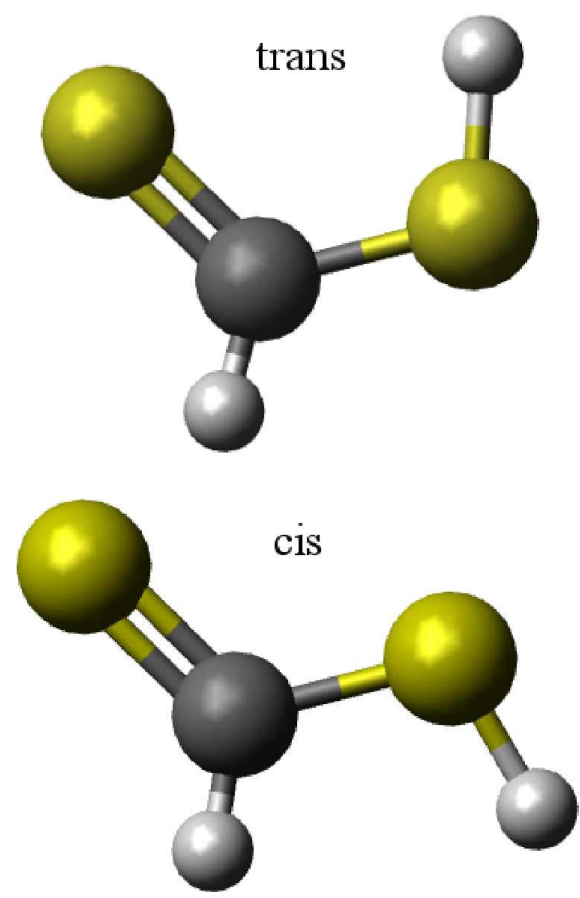

Fig. 1. Molecular structures of the two planar isomers of HCSSH. Details concerning the geometries of the two species are given in Sect. 3 and Appendix A. Yellow spheres indicate sulphur atoms, the dark grey sphere indicates carbon, and hydrogen atoms are shown in light grey.

expansion of the sulphur chemical network by the inclusion of these compounds and many other reactions and species (Woods et al. 2015; Vidal et al. 2017). Notwithstanding this progress, a number of important molecules are still not considered in the current picture of sulphur chemistry, including dithioformic acid, HCSSH. This species is chemically related to $\mathrm{CS}_{2}$ and is the S-bearing analogue of formic acid, $\mathrm{HCOOH}$; the latter was detected for the first time in SgrB2 by Zuckerman et al. (1971) and in the dark cloud L134N by Irvine et al. (1990). Because it is isostructural with $\mathrm{HCOOH}, \mathrm{HCSSH}$ exists in two different conformations, dubbed cis or trans, depending on the orientation of the $\mathrm{S}-\mathrm{H}$ bond with respect to the $\mathrm{C}-\mathrm{H}$ bond. These two conformers can be seen in Fig. 1.

The first laboratory study of dithioformic acid was carried out by Bak et al. (1978). They measured transitions in the centimetre $(\mathrm{cm})$ regime, between 18 and $40 \mathrm{GHz}$, for the trans and the cis conformers in the ground and in two lowlying vibrationally excited states. They also found an abundance ratio trans/cis $=5.43$, corresponding to an energy difference of $350 \mathrm{~cm}^{-1}$. The molecules were produced by gas-phase pyrolysis of methanetrithiol, $\mathrm{HC}(\mathrm{SH})_{3}$, and the spectra were recorded with a Stark-modulated microwave spectrometer. In a subsequent work, the same authors carried out additional measurements of the two ${ }^{34} \mathrm{~S}$ and one D isotopologues, leading to the experimental determination of the trans-HCSSH structure (Bak et al. 1979). However, the limited frequency range covered in these investigations prevented a satisfactory spectroscopic characterization of these species. In particular, the incomplete centrifugal distortion analysis and the rather high measurement errors caused by the interfering Stark lobes ${ }^{1}$ led to high estimated uncertainties in the spectral predictions. The calculated rest frequencies are, in fact,

\footnotetext{
1 Artefacts produced by the Stark modulation close to the centre frequency.
}

affected by errors exceeding $0.7 \mathrm{MHz}$ for the trans isomer and $1.1 \mathrm{MHz}$ for the cis, already in the $3 \mathrm{~mm}$ region.

Given the potential astronomical interest of this molecule, an improved knowledge of its rotational spectrum is desirable. In this work we present a thorough centrifugal distortion analysis of both trans and cis isomers of HCSSH. We extended the spectroscopic measurements well into the submillimetre (submm) domain, reaching a frequency as high as $478 \mathrm{GHz}$ for the transHCSSH. High-level theoretical calculations were also carried out to provide a detailed description of the molecular properties, such as dipole moment components and equilibrium structures.

\section{Experiments}

The measurements were performed using the CASAC (Center for Astrochemical Studies Absorption Cell) spectrometer at the Max-Planck-Institut für extraterrestrische Physik. Full details of the experimental apparatus can be found in Bizzocchi et al. (2017). The instrument employs the frequency modulation technique in the $\mathrm{mm}$ and submm range and is equipped with a glow discharge cell for production of unstable species.

Measurements were carried out at room temperature to avoid condensation of the precursors. Dithioformic acid was produced by a $40 \mathrm{~mA}$ DC discharge $(\sim 0.8 \mathrm{kV})$ from a 1:1 mixture of $\mathrm{CS}_{2}$ and $\mathrm{H}_{2}$, diluted in Ar buffer gas. The total pressure in the cell was 20-30 mTorr (27-40 $\mu$ bar).

\section{Results and data analysis}

The HCSSH isomers are near-prolate asymmetric rotors $\left(\kappa_{\text {trans }}=\right.$ -0.9901 and $\kappa_{\text {cis }}=-0.9682^{2}$ ) with $C_{\mathrm{s}}$ symmetry and planar structure. The a components of the dipole moment have been experimentally determined by Bak et al. (1978), where $\mu_{a}$ (trans) $=1.53 \mathrm{D}$ and $\mu_{a}$ (cis) $=2.10 \mathrm{D}$, while the $b$ components were determined in our study (see Appendix A). We measured 204 new line frequencies for the trans conformer and 139 for the cis. We also detected seven $b$-type transitions for the cis conformer, which has a higher value of $\mu_{b}$, equal to $1.67 \mathrm{D}$ (see Table A.2). These new rotational transitions belong to the $R$ branch $(\Delta J=+1)$ and $J$ values range from 14 to 74 and a maximum $K_{a}=20$.

We recovered the line central frequencies with the proFFit code (Dore 2003), adopting a modulated Voigt profile. The estimated accuracy ranges between $25-50 \mathrm{kHz}$, depending on the line width, signal-to-noise ratio, and background continuum (standing waves produced between the two partially reflecting windows placed on either side of the cell). Examples of measured line and fitted profile of trans and cis isomers are shown in Fig. 2. Both panels include lines corresponding to the blended $K_{a}=7$ asymmetry doublet, located at ca. $360 \mathrm{GHz}$.

We performed the spectral analysis using the Pickett SPFIT/SPCAT suit of programs (Pickett 1991), adopting the Watson S-reduced Hamiltonian for asymmetric-top molecules (Watson 1977). The transitions measured by Bak et al. (1978) in the $\mathrm{cm}$ range were also included. To take into account the different experimental accuracies, we employed four distinct statistical weights $\left(1 / \sigma^{2}\right)$ for the two datasets. For the lines taken from literature, we assigned $\sigma$ values of $75 \mathrm{kHz}$ and $100 \mathrm{kHz}$ to the trans and the cis isomers, respectively. A few lines showing very large

2 Ray's asymmetry parameter. It is an indication of the asymmetry of the molecule and can take values between -1 , for a prolate symmetric top, and +1 , for an oblate symmetric top (Gordy \& Cook 1984). 

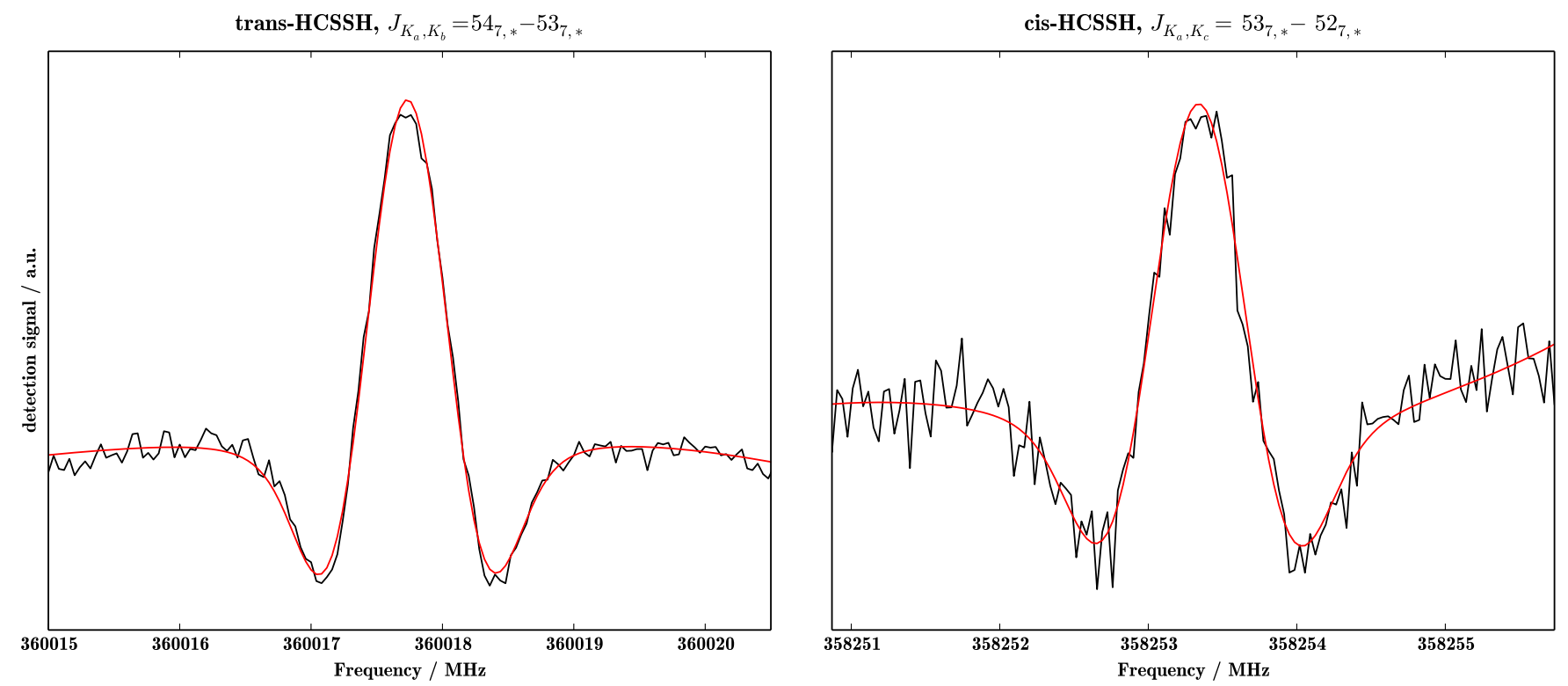

Fig. 2. Left panel: experimental spectra of the $54_{7, *}-53_{7, *}$ transitions of trans-HCSSH, with total integration time of $146 \mathrm{~s}$ and $3 \mathrm{~ms}$ of time constant. Right panel: experimental spectra of the $53_{7, *}-52_{7, *}$ transitions of cis-HCSSH with total integration time of $87 \mathrm{~s}$ and $3 \mathrm{~ms}$ of time constant. Both panels: The $K_{a}=7$ asymmetry doublet is not resolved. The red traces indicate the resulting line profile fitted with the proFFit code (see text).

deviation were removed from the final fit. Experimental uncertainties for our measurements are set to 25 or $50 \mathrm{kHz}$; the higher value is assigned only to lines broadened due to partially overlapped $K$ doublets. The final fits lead to the determination of the complete set of quartic centrifugal distortion constants and four sextic constants, i.e. $H_{J}, H_{J K}, H_{K J}$, and $h_{1}$ (energy contributions depending on $J^{6}$, obtained from the rotational Hamiltonian). One octic constant, i.e. $L_{K K J}$, was also determined for the trans isomer. The weighted root mean square (RMS) deviations are 0.936 and 0.933 for trans and cis, respectively. The resulting parameters are listed in Table 1. The lists of all the experimental frequencies are available at the CDS as supplementary data. Planarity of the species was confirmed by calculation of the inertial defect, $\Delta=I_{c}-\left(I_{b}+I_{a}\right)$ (Darling \& Dennison 1940), obtained using the moments of inertia $I_{\alpha}$, derived from the ground-state rotational constants. The inertial defect values for trans and cis dithioformic acid are 0.0185 and 0.0173 amu $\AA^{2}$, respectively, close to those of the two formic acid planar isomers, i.e. 0.0126 and 0.0102 amu $\AA^{2}$ (Trambarulo et al. 1958, Davis et al. 1980 and references therein, Winnewisser et al. 2002).

The complete line catalogues, computed by using the spectroscopic parameters in Table 1, are provided as supplementary data at the CDS and also include the $1 \sigma$ uncertainties, upperstate energies, line strengths, and the Einstein $A$ coefficients for spontaneous emission, all expressed in SI units as follows:

$A_{i j}=\frac{16 \pi^{3} v^{3}}{3 \epsilon_{0} h c^{3}} \frac{1}{2 J+1} S_{i j} \mu^{2}$

where $v$ is the line frequency, $\epsilon_{0}$ is the vacuum permittivity, $h$ is the Plank's constant, $c$ is the speed of light, $J$ is the quantum number of the upper state for the rotational angular momentum, and $S_{i j} \mu^{2}$ is the line strength. The lists consist of transitions with $E_{u} / k<300 \mathrm{~K}$ and predicted uncertainties lower than $50 \mathrm{kHz}$, resulting in lines between 0.2 and $300 \mathrm{GHz}$. All of these rest frequencies have at least a precision of $6 \times 10^{-8}$, corresponding to a radial velocity of $0.018 \mathrm{~km} \mathrm{~s}^{-1}$ or even better. Partition functions at various temperatures for both isomers are listed in Table 2.

\section{Discussion and conclusions}

This laboratory study provides a comprehensive spectroscopic characterization of the two stable conformers of HCSSH in the ground state. Extension of the measurements up to $478 \mathrm{GHz}$ and the resulting larger dataset lead to an overall improvement of the spectroscopic parameters with respect to that of Bak et al. (1978). In particular, the uncertainties on the $A$ rotational constant and on the $D_{J}$ centrifugal distortion constant were reduced by more than 3 orders of magnitude. We extended the centrifugal distortion analysis with all the quartic and four sextic constants. Additionally, the octic $L_{K K J}$ parameter was included for the trans conformer. Further improvements in the fit of the cis conformer were obtained through measurements of $b$-type transitions. These new sets of spectroscopic parameters made it possible to compute accurate rest frequencies. In comparison to previous studies, predicted uncertainties were significantly reduced and have values as low as $2 \mathrm{kHz}\left(0.006 \mathrm{~km} \mathrm{~s}^{-1}\right)$ at $3 \mathrm{~mm}$ wavelength.

An astronomical search of these molecules can be carried out in the $\mathrm{cm}, \mathrm{mm}$, and submm bands, depending on the temperature of the source. As shown in Fig. 3, in cold environments with $T_{\text {kin }} \sim 10 \mathrm{~K}$ or lower (such as the S-rich prestellar cores L183 and Barnard 1), assuming local thermal equilibrium, calculated line intensities and distribution for the trans isomer peak around $45 \mathrm{GHz}$. In warm regions with $T_{\text {kin }} \sim 100 \mathrm{~K}$, the peak shifts at higher frequencies, ca. $200 \mathrm{GHz}$. Owing to its larger $b$ component of the dipole moment, the cis isomer shows a different intensity distribution. In this case the calculated peak lies at $\sim 280 \mathrm{GHz}$ in cold sources and at $\sim 780 \mathrm{GHz}$ in warm regions. However, the strongest lines above $300 \mathrm{GHz}$, i.e. $b$-type transitions with low $E_{u} / k$, present uncertainties higher than $1 \mathrm{MHz}$. These large errors are a consequence of the restricted number of measurable $b$-type transitions, strongly limited by the overall lower intensity of the cis' lines with respect to the trans.

Work is also under way in our group to extend astrochemical modelling of sulphur to a number of new species, including HCSSH (Laas et al., in prep.). Although this molecule has not 
Table 1. Spectroscopic parameters of the two conformers of HCSSH.

\begin{tabular}{llcccc}
\hline \hline Constants & Units & trans-HCSSH $^{a}$ & trans-HCSSH $^{b}$ & cis-HCSSH $^{a}$ & cis-HCSSH $^{b}$ \\
\hline$A_{0}$ & $\mathrm{MHz}$ & $49206.11(21)^{c}$ & $49227 .(86)$ & $48572.439(66)$ & $48947 .(380)$ \\
$B_{0}$ & $\mathrm{MHz}$ & $3447.53432(37)$ & $3447.5312(89)$ & $3498.74789(67)$ & $3498.719(24)$ \\
$C_{0}$ & $\mathrm{MHz}$ & $3219.47256(37)$ & $3219.4954(96)$ & $3261.42278(62)$ & $3261.433(30)$ \\
$D_{J}$ & $\mathrm{kHz}$ & $1.063389(64)$ & $1.10(10)^{d}$ & $1.27724(14)$ & $1.02(42)^{d}$ \\
$D_{J K}$ & $\mathrm{MHz}$ & $-0.0389438(19)$ & $-0.03905(24)^{d}$ & $-0.0470807(33)$ & $-0.0484(12)^{d}$ \\
$D_{K}$ & $\mathrm{MHz}$ & $1.376(33)$ & & $1.412(17)$ & \\
$d_{1}$ & $\mathrm{kHz}$ & $-0.119787(80)$ & & $-0.14900(20)$ & \\
$d_{2}$ & $\mathrm{~Hz}$ & $-3.609(38)$ & & $-4.343(28)$ & \\
$H_{J}$ & $\mathrm{~Hz}$ & $0.0005714(64)$ & & $0.001169(25)$ & \\
$H_{J K}$ & $\mathrm{~Hz}$ & $-0.00573(31)$ & & $-0.03601(50)$ & \\
$H_{K J}$ & $\mathrm{~Hz}$ & $-4.216(15)$ & & $-4.109(20)$ & \\
$h_{1}$ & $\mathrm{~Hz}$ & $0.0001673(96)$ & & $0.000288(38)$ & \\
$L_{K K J}$ & $\mathrm{~Hz}$ & $0.000447(27)$ & & & \\
$I_{c}-\left(I_{b}+I_{a}\right)$ & $\mathrm{amu} \mathrm{A}$ & 0.0185 & & 0.0173 & \\
$\sigma_{w}$ & & 0.936 & & 0.933 & 139 \\
No. of lines & \multicolumn{7}{c}{204} & 25 & & \\
\hline
\end{tabular}

Notes. The results of this study are compared with those of Bak et al. (1978). ${ }^{(a)}$ This work. ${ }^{(b)}$ Bak et al. (1978). ${ }^{(c)}$ Standard error in parentheses are in units of the last digit. ${ }^{(d)}$ Calculated using the Watson A-reduction for asymmetric top molecules.
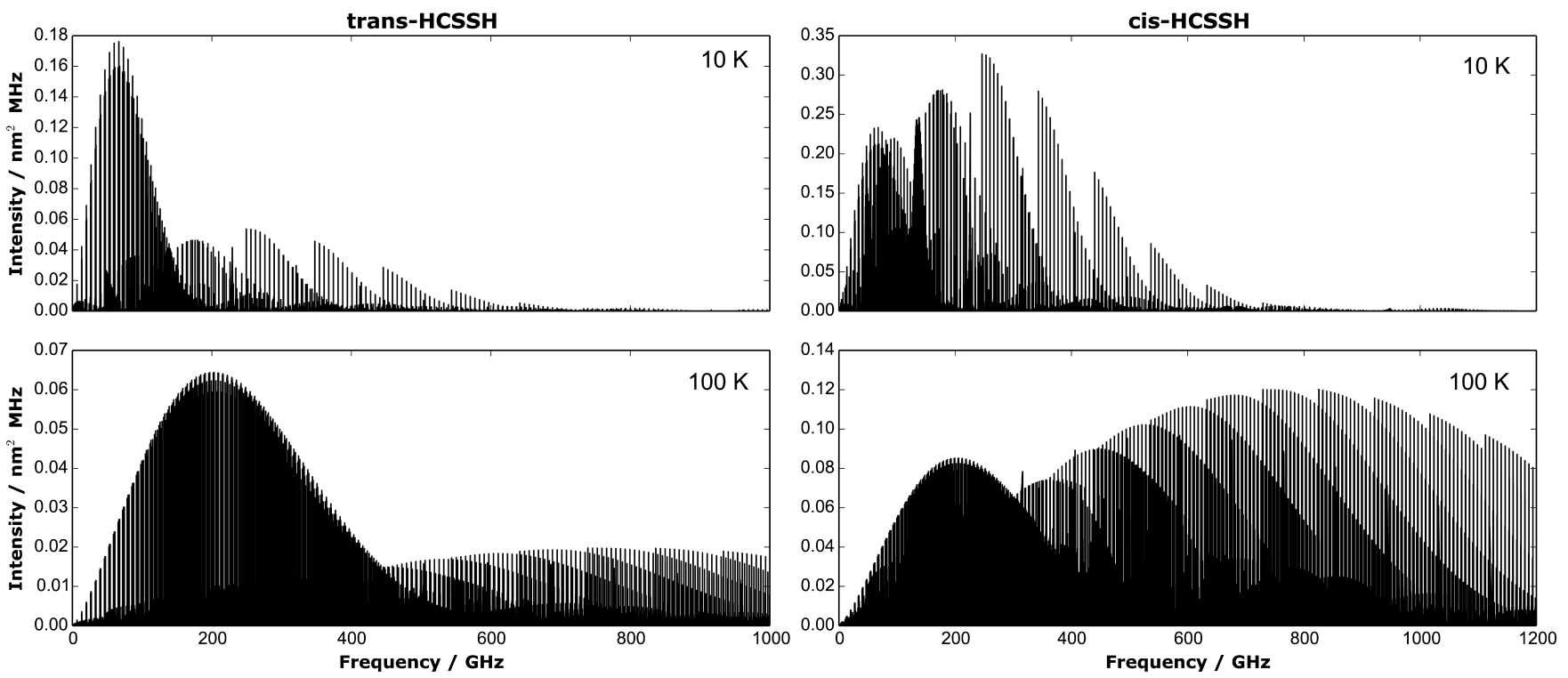

Fig. 3. Intensities and line distributions of rotational transitions at 10 and $100 \mathrm{~K}$, for trans- and cis-dithioformic acid isomers. Most of the bandheads above $250 \mathrm{GHz}$ are produced by $b$-type transitions.

been previously studied in the context of astrochemical modelling, a number of reactions may be readily tested, based on standard grain surface rates (Hasegawa et al. 1992) or ionneutral gas-phase kinetics (Troe 1985). Analogous to many other interstellar complex organic molecules, it is likely that a series of barrierless radical-radical addition reactions might lead to its formation on grain surfaces, such as

$\mathrm{HS}+\mathrm{CS} \rightarrow \mathrm{CSSH}$,

$\mathrm{CSSH}+\mathrm{H} \rightarrow \mathrm{HCSSH}$,

in analogy with the efficient formation of the O-bearing counterpart formic acid. In the gas phase, $\mathrm{HCOOH}$ may be efficiently formed via the ionic route,

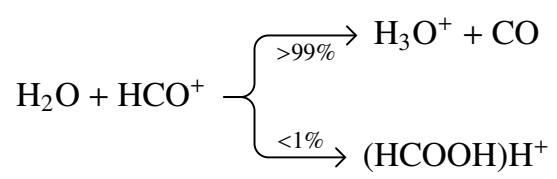

$$
(\mathrm{HCOOH}) \mathrm{H}^{+}+\mathrm{e}^{-} \rightarrow \mathrm{HCOOH}+\mathrm{H},
$$

and the analogue of this route will also be tested for the gasphase formation of HCSSH. If HCSSH is indeed found to be formed efficiently in a way similar to $\mathrm{HCOOH}$, it is likely that the most promising sources for detection are S-rich regions, where formic acid can be routinely observed. 
Table 2. Partition functions of trans-HCSSH and cis-HCSSH at various temperatures.

\begin{tabular}{ccc}
\hline \hline$T(\mathrm{~K})$ & $Q$ (trans-HCSSH) & $Q$ (cis-HCSSH $)$ \\
\hline 300 & 37126.4660 & 36887.4922 \\
200 & 20421.8656 & 20276.8962 \\
100 & 7224.8408 & 7172.3000 \\
50 & 2554.3700 & 2535.7348 \\
20 & 647.0112 & 642.2926 \\
10 & 229.3211 & 227.6550 \\
\hline
\end{tabular}

Acknowledgement. The authors wish to thank L. Dore for providing the proFFit code.

\section{References}

Bak, B., Nielsen, O. J., \& Svanholt, H. 1978, J. Mol. Spectr., 69, 401 Bak, B., Nielsen, O., Svanholt, H., \& Christiansen, J. J. 1979, J. Mol. Spectr., 75, 134

Bizzocchi, L., Lattanzi, V., Laas, J., et al. 2017, A\&A, 602, A34

Calmonte, U., Altwegg, K., Balsiger, H., et al. 2016, MNRAS, 462, S253

Darling, B. T., \& Dennison, D. M. 1940, Phys. Rev., 57, 128

Davis, R. W., Robiette, A., Gerry, M., Bjarnov, E., \& Winnewisser, G. 1980, J. Mol. Spectr., 81, 93

Deegan, M. J., \& Knowles, P. J. 1994, Chem. Phys. Lett., 227, 321

Dore, L. 2003, J. Mol. Spectr., 221, 93

Druard, C., \& Wakelam, V. 2012, MNRAS, 426, 354

Dunning Jr, T. H. 1989, J. Chem. Phys., 90, 1007

Dunning Jr, T. H., Peterson, K. A., \& Wilson, A. K. 2001, J. Chem. Phys., 114, 9244

Feller, D. 1992, J. Chem. Phys., 96, 6104

Feller, D. 1993, J. Chem. Phys., 98, 7059

Ferrante, R. F., Moore, M. H., Spiliotis, M. M., \& Hudson, R. L. 2008, ApJ, 684, 1210

Garozzo, M., Fulvio, D., Kanuchova, Z., Palumbo, M. E., \& Strazzulla, G. 2010, A\&A, 509, A67

Gordy, W., \& Cook, R. L. 1984, Microwave Molecular Spectra (Wiley)

Halkier, A., Klopper, W., Helgaker, T., \& Jørgensen, P. 1999, J. Chem. Phys., 111,4424

Hampel, C., Peterson, K. A., \& Werner, H.-J. 1992, Chem. Phys. Lett., 190, 1

Hasegawa, T. I., Herbst, E., \& Leung, C. M. 1992, ApJS, 82, 167

Heckert, M., Kállay, M., Tew, D. P., Klopper, W., \& Gauss, J. 2006, J. Chem. Phys., 125, 044108
Hehre, W. J. 1986, Ab initio Molecular Orbital Theory (Wiley-Interscience)

Helgaker, T., Klopper, W., Koch, H., \& Noga, J. 1997, J. Chem. Phys., 106, 9639

Irvine, W. M., Friberg, P., Kaifu, N., et al. 1990, A\&A, 229, L9

Jackson, W. M., Scodinu, A., Xu, D., \& Cochran, A. L. 2004, ApJ, 607, L139

Jefferts, K. B., Penzias, A. A., Wilson, R. W., \& Solomon, P. M. 1971, ApJ, 168, L111

Jenkins, E. B. 1987, Interstellar Processes (Springer), 134, 533

Jiménez-Escobar, A., \& Muñoz Caro, G. M. 2011, A\&A, 536, A91

Jiménez-Escobar, A., Muñoz Caro, G. M., \& Chen, Y.-J. 2014, MNRAS, 443, 343

Kendall, R. A., Dunning Jr, T. H., \& Harrison, R. J. 1992, J. Chem. Phys., 96, 6796

Kolesniková, L., Tercero, B., Cernicharo, J., et al. 2014, ApJ, 784, L7

Krishnan, R., \& Pople, J. A. 1978, Int. J. Quant. Chem., 14, 91

Linke, R. A., Frerking, M. A., \& Thaddeus, P. 1979, ApJ, 234, L139

Møller, C., \& Plesset, M. S. 1934, Phys. Rev., 46, 618

Nguyen, M. T., Nguyen, T. L., \& Le, H. T. 1999, J. Phys. Chem. A, 103, 5758

Oppenheimer, M., \& Dalgarno, A. 1974, ApJ, 187, 231

Penzias, A. A., Solomon, P. M., Wilson, R. W., \& Jefferts, K. B. 1971, ApJ, 168, L53

Peterson, K. A., \& Dunning Jr, T. H. 2002, J. Chem. Phys., 117, 10548

Pickett, H. M. 1991, J. Mol. Spectr., 148, 371

Pople, J. A., Binkley, J. S., \& Seeger, R. 1976, Int. J. Quant. Chem., 10, 1

Pople, J. A., Head-Gordon, M., \& Raghavachari, K. 1987, J. Chem. Phys., 87, 5968

Purvis III, G. D., \& Bartlett, R. J. 1982, J. Chem. Phys., 76, 1910

Raghavachari, K., Trucks, G. W., Pople, J. A., \& Head-Gordon, M. 1989, Chem. Phys. Lett., 157, 479

Ruffle, D., Hartquist, T., Caselli, P., \& Williams, D. 1999, MNRAS, 306, 691

Savage, B. D., \& Sembach, K. R. 1996, Annu. Rev. Astron. Astrophys., 34, 279

Sinclair, M. W., Fourikis, N., Ribes, J. C., et al. 1973, Aust. J. Phys., 26, 85

Thaddeus, P., Kutner, M. L., Penzias, A. A., Wilson, R. W., \& Jefferts, K. B. 1972, ApJ, 176, L73

Tieftrunk, A., Pineau des Forets, G., Schilke, P., \& Walmsley, C. M. 1994, A\&A, 289,579

Trambarulo, R., Clark, A., \& Hearns, C. 1958, J. Chem. Phys., 28, 736

Troe, J. 1985, Chem. Phys. Lett., 122, 425

Vidal, T. H., Loison, J.-C., Jaziri, A. Y., et al. 2017, MNRAS, 469, 435

Wakelam, V., \& Herbst, E. 2008, ApJ, 680, 371

Wakelam, V., Caselli, P., Ceccarelli, C., Herbst, E., \& Castets, A. 2004, A\&A 422, 159

Wakelam, V., Caselli, P., Ceccarelli, C., et al. 2005, in ESA SP, 577, ed. A. Wilson, 435

Watson, J.K. G. 1977, in Vibrational Spectra and Structure, ed. J. Durig (Amsterdam: Elsevier), 6, 1

Winnewisser, M., Winnewisser, B. P., Stein, M., et al. 2002, J. Mol. Spectr., 216, 259

Woods, P. M., Occhiogrosso, A., Viti, S., et al. 2015, MNRAS, 450, 1256

Zuckerman, B., Ball, J. A., \& Gottlieb, C. A. 1971, ApJ, 163, L41 


\section{Appendix A: Theoretical calculations}

Dithioformic acid has been the subject of numerous ab initio calculations in the past in which structures and conformational behaviour, isomerization, unimolecular rearrangement and decomposition reaction paths have been studied (Nguyen et al. 1999 and references therein). Apart from Nguyen et al. (1999), who made use of QCISD(T) methods (Pople et al. 1987), the older studies had been carried out at the HF-SCF (Hehre 1986) or MP2 (Møller \& Plesset 1934; Pople et al. 1976; Krishnan \& Pople 1978) levels, also making use of small basis sets and thus leading to less accurate results. In our work, high level quantum-chemical calculations were performed, focussing on an accurate estimation of dipole moments and equilibrium structures.

Geometry optimization was carried out via the $\operatorname{CCSD}(\mathrm{T})$ method (Purvis III \& Bartlett 1982; Raghavachari et al. 1989; Hampel et al. 1992; Deegan \& Knowles 1994). Employing the frozen-core approximation, we used the correlation consistent valence basis sets cc-pVnZ ( $n=T, Q, 5)$ (Dunning Jr 1989) for the hydrogen and carbon atoms and the $d$-augmented basis sets cc-pV(n+d)Z (Dunning Jr et al. 2001) for the sulphur atoms. We also provide an estimate of the equilibrium energies based on a hierarchical sequence of basis sets, using the complete basis set (CBS) limit extrapolation, as described in Heckert et al. (2006). The molecules have been considered as planar because of the assumption based on the inertial defect (see Sect. 3). The total energies have been obtained as a sum of the Hartree-Fock self-consistent field (HF-SCF) energy, the electron-correlation contribution at the $\operatorname{CCSD}(\mathrm{T})$ level, and the core-valence (CV) correlation effect. CBS limit extrapolation has been applied for the first two values as follows:

$E_{\text {tot }}=E_{\infty}^{\mathrm{HF}-\mathrm{SCF}}+\Delta E_{\infty}^{\mathrm{CCSD}(\mathrm{T})}+\Delta E_{\mathrm{CV}}$.

The convergence of the Hartree-Fock CBS limit has been evaluated with (Feller 1992, 1993)

$E^{\mathrm{HF}-\mathrm{SCF}}(n)=E_{\infty}^{\mathrm{HF}-\mathrm{SCF}}+A e^{-B n}$, while the function used to extrapolate the value of electroncorrelation energy to the CBS limit is (Helgaker et al. 1997) written as

$\Delta E^{\mathrm{CCSD}(\mathrm{T})}(n)=\Delta E_{\infty}^{\mathrm{CCSD}(\mathrm{T})}+C n^{-3}$.

The core-valence correction has been calculated and added as follows:

$\Delta E_{\mathrm{CV}}=E_{a e}-E_{f c}$,

where both the all electron $\left(E_{a e}\right)$ and the frozen-core $\left(E_{f c}\right)$ energies have been calculated using the cc-pwCVQZ basis set (Peterson \& Dunning Jr 2002), avoiding correlation of 1s electrons of sulphur in any computation. All the calculations have been performed with the CFOUR program package ${ }^{3}$.

The results show that the trans conformer is the most stable and has a difference in energy of $421 \mathrm{~cm}^{-1}$ with respect to the cis-HCSSH. This value is in reasonable agreement with the experimental value given by Bak et al. (1978), which differs only by $71 \mathrm{~cm}^{-1}$. Molecular structures were obtained with the same method shown above. All the results are listed in Table A.1.

Dipole moments were also calculated and extrapolated to the CBS limit with a similar procedure (Halkier et al. 1999). For these dipole calculations, we used similar basis sets, but augmented with diffuse functions to ensure correct evaluation of this property: that is, the light atoms used the aug-cc-pVnZ basis sets (Kendall et al. 1992) and the sulphur atoms used the aug-cc-pV(n+d)Z basis sets (Dunning Jr et al. 2001). The computations were carried out using the CBS + CV equilibrium geometries, i.e. the best estimated structure obtained with the additivity assumption from Eq. (A.1). The final values are shown in Table A.2. Owing to its higher computational cost, the barrier energy to trans - cis conversion was computed at the $\operatorname{CCSD}(\mathrm{T}) / \mathrm{cc}-\mathrm{pVTZ}$ level, with the frozen-core approximation, giving a value of $3762 \mathrm{~cm}^{-1}$.

3 wWW. cfour.de 
Table A.1. Equilibrium geometries and energies of trans-HCSSH and cis-HCSSH are reported.

\begin{tabular}{lllllllll}
\hline \hline Basis set & Energy (Hartree) & $\mathrm{C}=\mathrm{S}(\AA)$ & $\mathrm{C}-\mathrm{S}(\AA)$ & $\mathrm{S}-\mathrm{H}(\AA)$ & $\mathrm{C}-\mathrm{H}(\AA)$ & $L^{a} \mathrm{~S}=\mathrm{C}-\mathrm{S}\left({ }^{\circ}\right)$ & $\angle \mathrm{C}-\mathrm{S}-\mathrm{H}\left(^{\circ}\right)$ & $\angle \mathrm{H}-\mathrm{C}=\mathrm{S}\left({ }^{\circ}\right)$ \\
\hline trans-HCSSH & & & & & & & & \\
\hline cc-pVTZ & -834.692586 & 1.6259 & 1.7412 & 1.3418 & 1.0896 & 128.27 & 95.49 & 110.23 \\
cc-pVQZ & -834.739631 & 1.6223 & 1.7352 & 1.3421 & 1.0891 & 128.09 & 95.61 & 110.43 \\
cc-pV5Z & -834.754180 & 1.6212 & 1.7332 & 1.3420 & 1.0891 & 128.04 & 95.64 & 110.50 \\
CBS $^{b}$ & -834.767911 & 1.6201 & 1.7314 & 1.3420 & 1.0891 & 127.98 & 95.66 & 110.54 \\
cc-pwCVQZ(fc) $^{\circ}$ & -834.743529 & 1.6214 & 1.7341 & 1.3415 & 1.0891 & 128.09 & 95.61 & 110.44 \\
cc-pwCVQZ(ae) & -835.433398 & 1.6173 & 1.7297 & 1.3394 & 1.0877 & 128.11 & 95.61 & 110.44 \\
CBS+CV & -835.457780 & 1.6161 & 1.7270 & 1.3398 & 1.0877 & 127.99 & 95.66 & 110.54 \\
\hline cis-HCSSH & & & & & & & & \\
cc-pVTZ & -834.690500 & 1.6247 & 1.7454 & 1.3412 & 1.0881 & 123.75 & 96.59 & 114.16 \\
cc-pVQZ & -834.737690 & 1.6211 & 1.7391 & 1.3413 & 1.0877 & 123.58 & 96.79 & 114.33 \\
cc-pV5Z & -834.752258 & 1.6200 & 1.7370 & 1.3412 & 1.0877 & 123.52 & 96.83 & 114.39 \\
CBS & -834.765988 & 1.6189 & 1.7352 & 1.3411 & 1.0877 & 123.46 & 96.86 & 114.45 \\
cc-pwCVQZ(fc) & -834.741583 & 1.6202 & 1.7380 & 1.3407 & 1.0877 & 123.56 & 96.80 & 114.34 \\
cc-pwCVQZ(ae) & -835.431447 & 1.6161 & 1.7336 & 1.3385 & 1.0862 & 123.55 & 96.82 & 114.37 \\
CBS+CV & -835.455852 & 1.6149 & 1.7309 & 1.3389 & 1.0862 & 123.45 & 96.88 & 114.48 \\
\hline
\end{tabular}

Notes. All the values were computed at the $\operatorname{CCSD}(\mathrm{T})$ level of theory. ${ }^{(a)}$ Bond angle in degree. ${ }^{(b)} \mathrm{CBS}$ extrapolation using the additivity assumption shown in Eq. (A.1) without core-valence correction, $\Delta E_{C V}$. ${ }^{(c)}$ Final best estimated values, calculated using the complete additivity assumption shown in Eq. (A.1).

Table A.2. Dipole moments of trans-HCSSH and cis-HCSSH.

\begin{tabular}{lcc}
\hline \hline Basis set & $\mu_{a}$ (Debye) & $\mu_{b}$ (Debye) \\
\hline trans-HCSSH & & \\
\hline aug-cc-pVTZ & 1.4788 & 0.1950 \\
aug-cc-pVQZ & 1.4870 & 0.1957 \\
aug-cc-pV5Z & 1.4807 & 0.1939 \\
CBS $^{a}$ & 1.4800 & 0.1935 \\
aug-cc-pCVQZ(fc) & 1.4863 & 0.1945 \\
aug-cc-pCVQZ(ae) & 1.4822 & 0.1934 \\
CBS+CV & 1.4766 & 0.1924 \\
\hline cis-HCSSH $^{b}$ & & \\
aug-cc-pVTZ & 2.0756 & 1.6323 \\
aug-cc-pVQZ & 2.0896 & 1.6474 \\
aug-cc-pV5Z & 2.0878 & 1.6448 \\
CBS $^{a}$ & 2.0861 & 1.6428 \\
aug-cc-pwCVQZ(fc) & 2.0883 & 1.6489 \\
aug-cc-pwCVQZ(ae) & 2.0834 & 1.6464 \\
CBS+CV $^{b}$ & 2.0829 & 1.6403 \\
\hline
\end{tabular}

Notes. All the values were computed at the $\operatorname{CCSD}(\mathrm{T})$ level of theory. (a) $\mathrm{CBS}$ extrapolation using the additivity assumption shown in Eq. (A.1) without core-valence correction, $\Delta E_{C V}{ }^{\left({ }^{(b)}\right.}$ Final best estimated values, calculated using the complete additivity assumption shown in Eq. (A.1). 